[Supporting Information]

\title{
Lithium Salt-induced Microstructure and Ordering in Diblock Copolymer/Homopolymer Blends
}

Matthew T. Irwin, ${ }^{1, a}$ Robert J. Hickey, ${ }^{2, a}$ Shuyi Xie, ${ }^{2}$ Frank S. Bates, ${ }^{1}$ and Timothy P. Lodge*,1,2

${ }^{1}$ Department of Chemical Engineering and Materials Science and ${ }^{2}$ Department of Chemistry, University of Minnesota, Minneapolis, MN 55455

a.) Authors contributed equally to this work

Authors for correspondence: lodge@umn.edu, bates001@umn.edu 


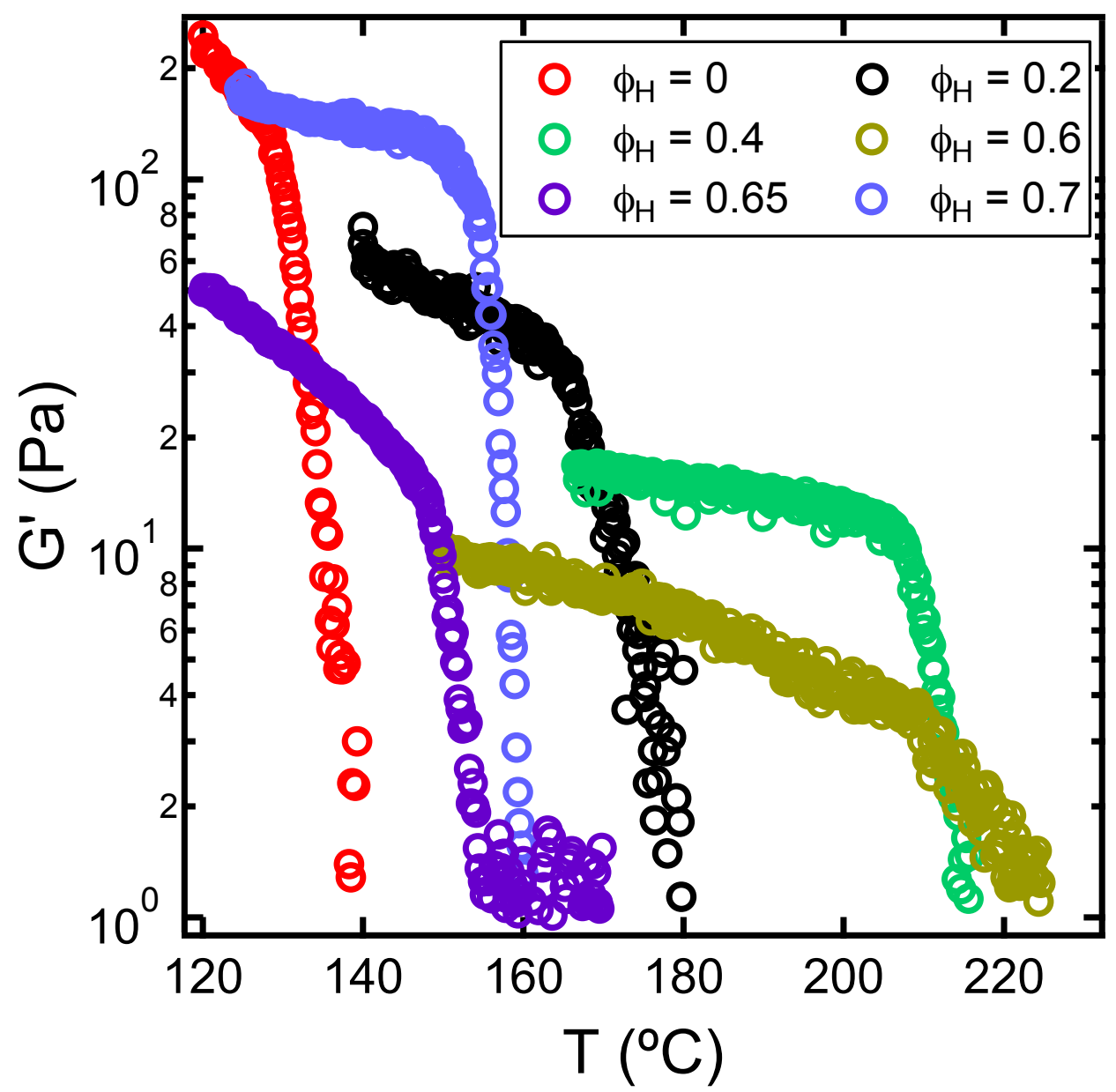

Figure S1. Elastic modulus for various blend compositions at a frequency of $1 \mathrm{rad} / \mathrm{s}$ upon heating at $1{ }^{\circ} \mathrm{C} / \mathrm{min}$. The sudden drop in the elastic modulus at a particular temperature is a signature of the order-disorder transition. 


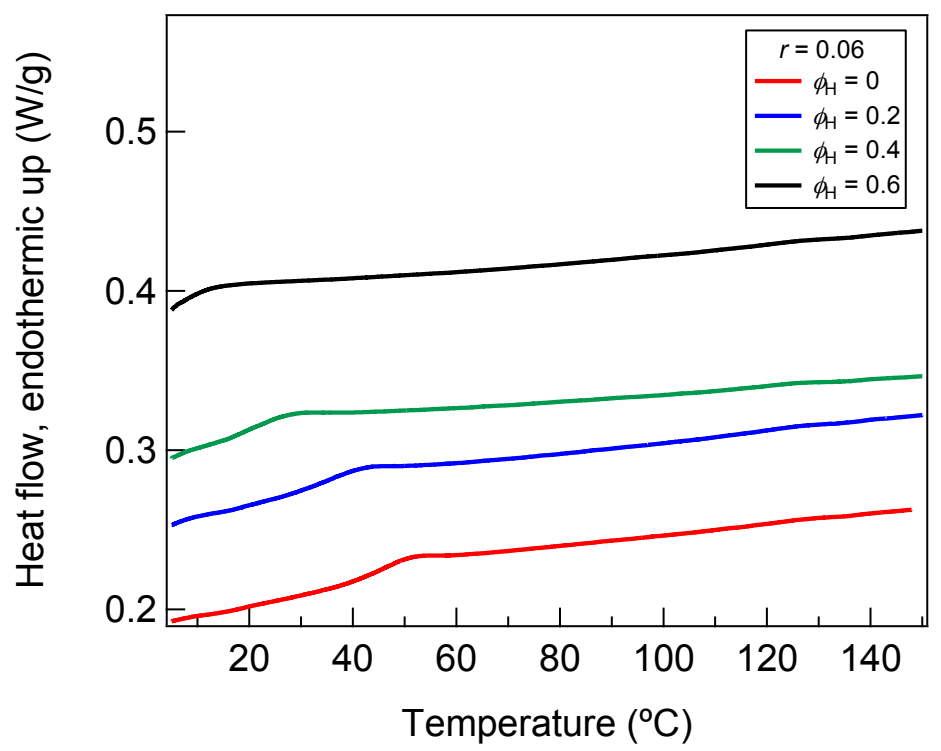

Figure S2. DSC thermograms for four salt-containing blends. Traces have been offset vertically by multiples of $0.05 \mathrm{~W} / \mathrm{g}$. Data shown are from a second heating at $10{ }^{\circ} \mathrm{C} / \mathrm{min}$. There is no observable crystallinity in the blends at room temperature. The data also indicate that the glass transition temperature of the PS domain is depressed with increasing homopolymer loading, concurrent with a decrease in the average molecular weight in the PS domain. 


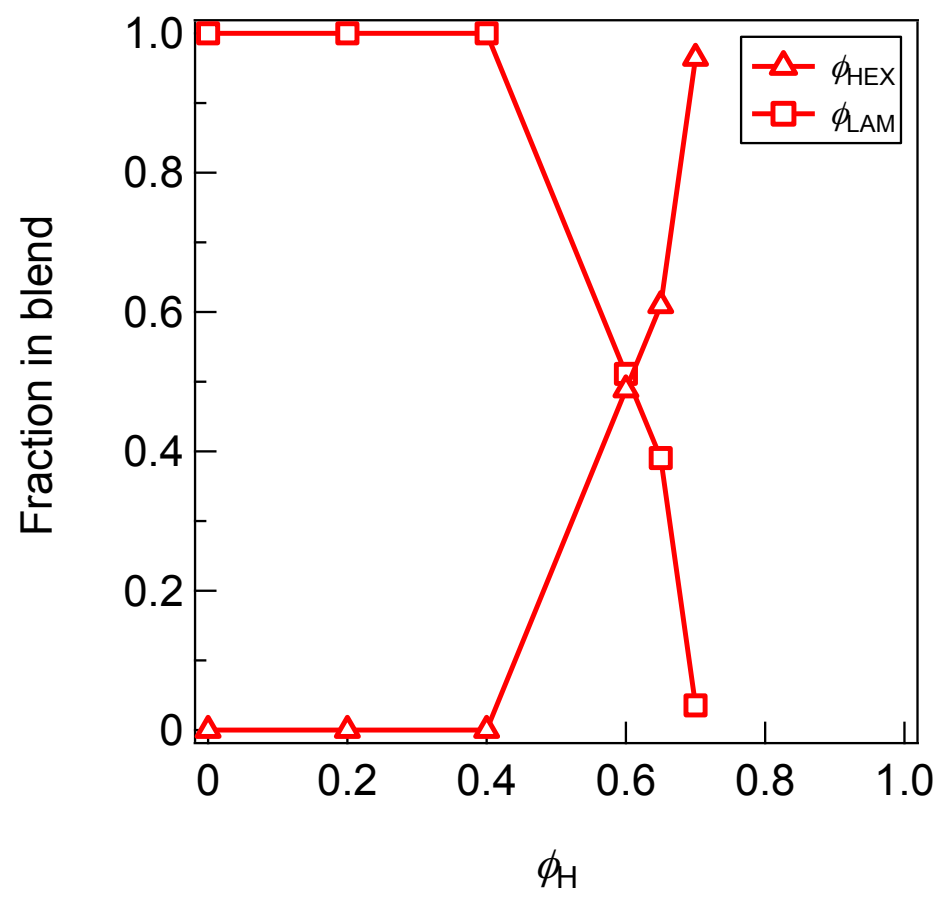

Figure S3. Comparison of the relative fraction of LAM and HEX of blends at $120{ }^{\circ} \mathrm{C}$ as a function of the volume fraction of homopolymer. The relatively fraction of each ordered phase was estimated by comparing the relative intensities of the primary scattering peaks for the LAM and HEX morphologies as assessed from SAXS experiments. The lines are drawn to guide the eye. 


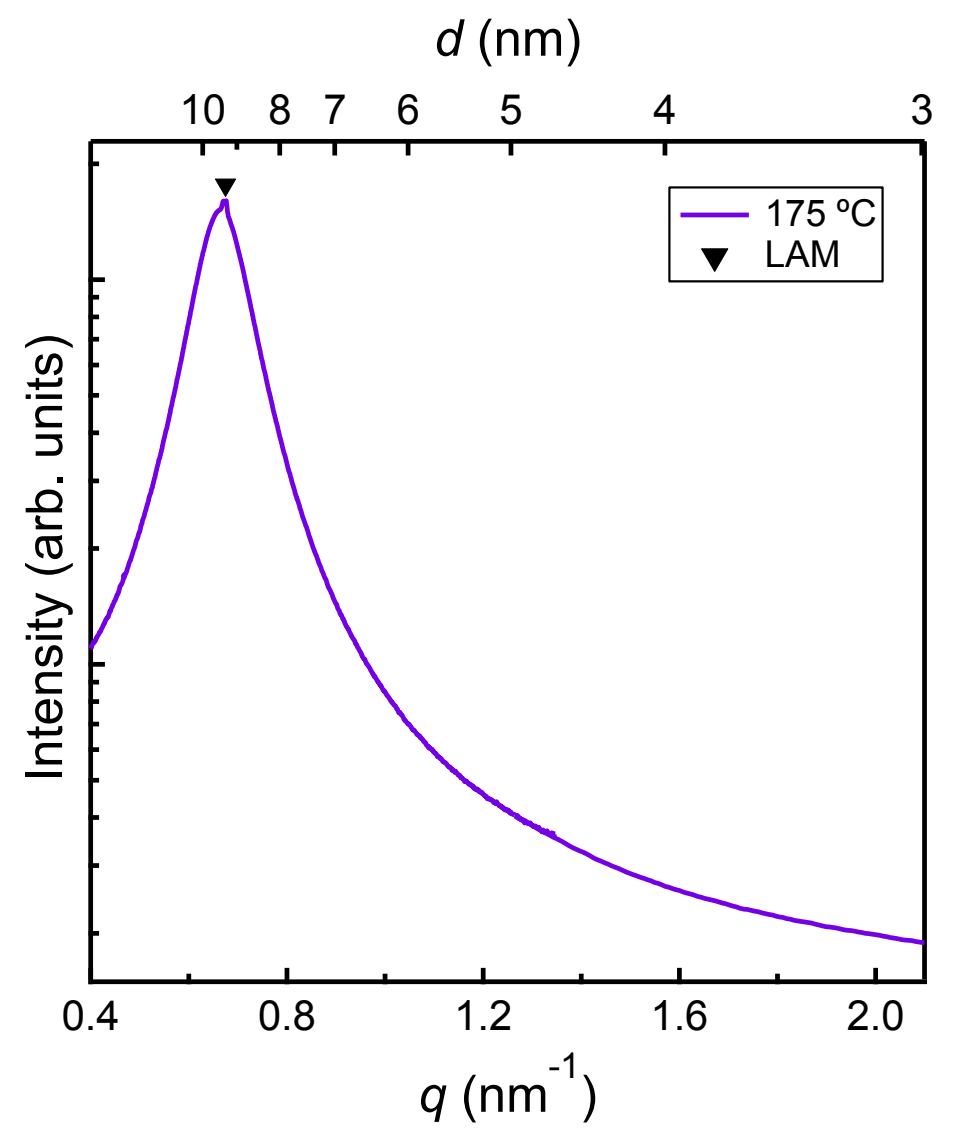

Figure S4. Small angle X-ray scattering trace for a blend containing $\phi_{\mathrm{H}}=0.2$ at $175{ }^{\circ} \mathrm{C}$. The marker indicates the presence of a slight, but observable, $q^{*}$ peak that suggests the coexistence of LAM and DIS phases. 


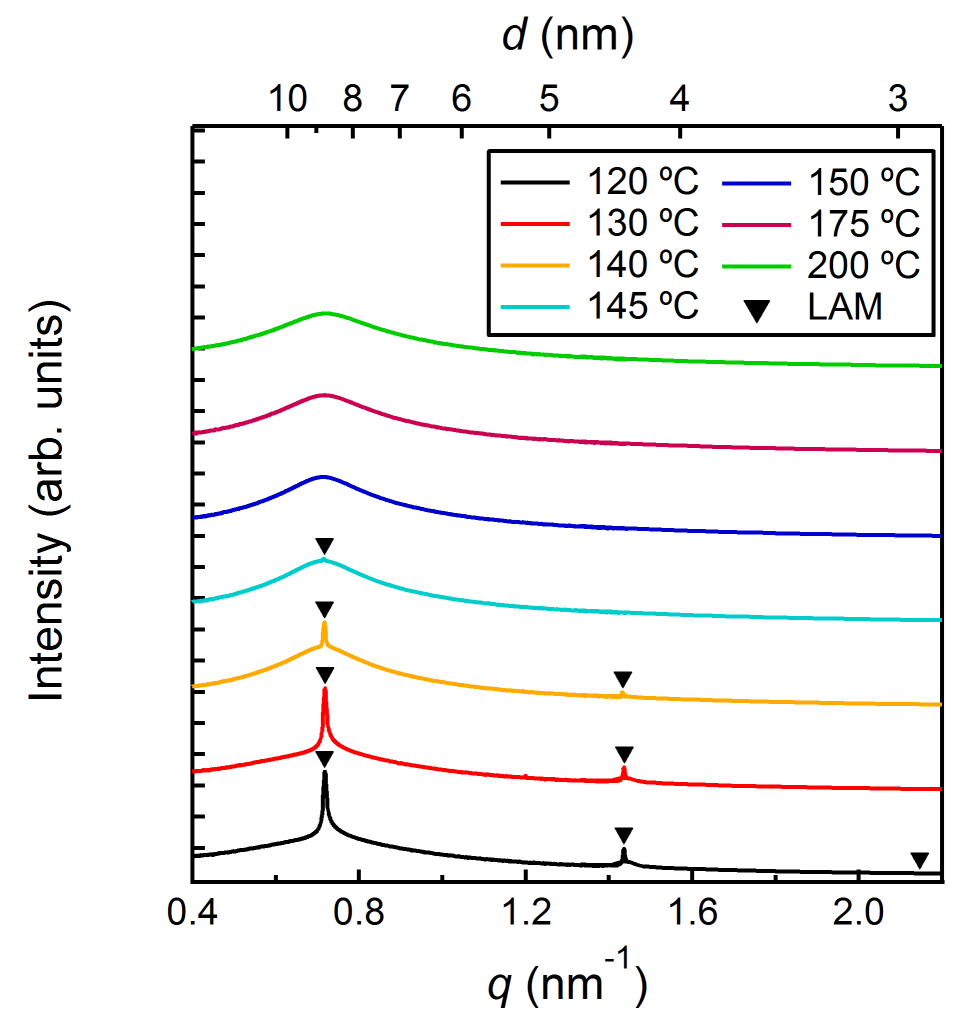

Figure S5. Small angle X-ray scattering traces for the block copolymer $\left(\phi_{\mathrm{H}}=0\right)$ doped with LiTFSI at a molar ratio $r=[\mathrm{Li}] /[\mathrm{EO}]=0.06$. Markers indicate $q / q^{*}$ locations of 1,2 , and 3, where $q^{*}$ is the primary peak position. 


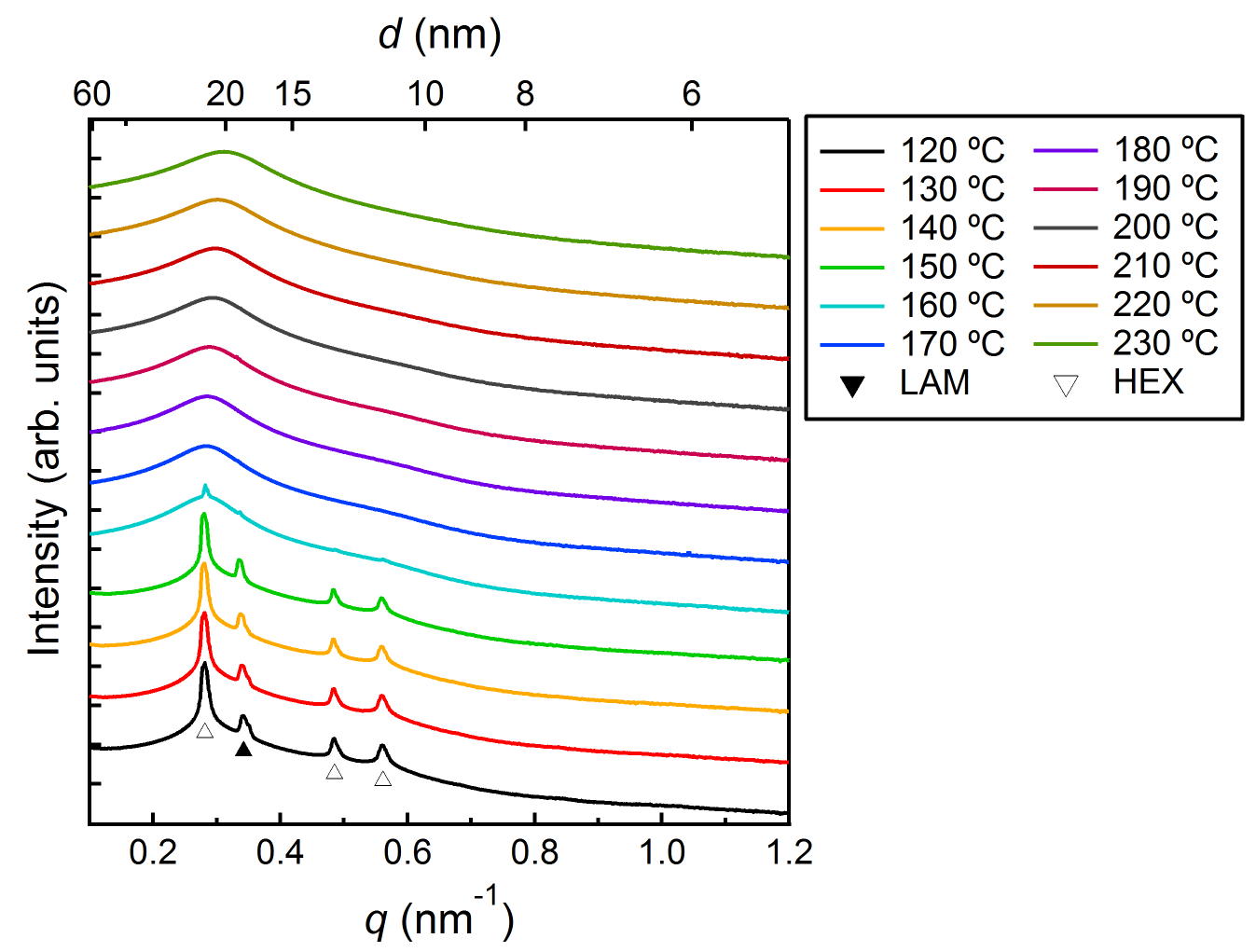

Figure S6. Small angle X-ray scattering traces for a blend containing $\phi_{\mathrm{H}}=0.7$ doped with LiTFSI at a molar ratio $r=[\mathrm{Li}] /[\mathrm{EO}]=0.06$. The LAM marker indicates only the primary peak, $q^{*}$, while the HEX markers indicate peak positions $q / q^{*}$ of $1,3^{1 / 2}$, and $4^{1 / 2}$. 


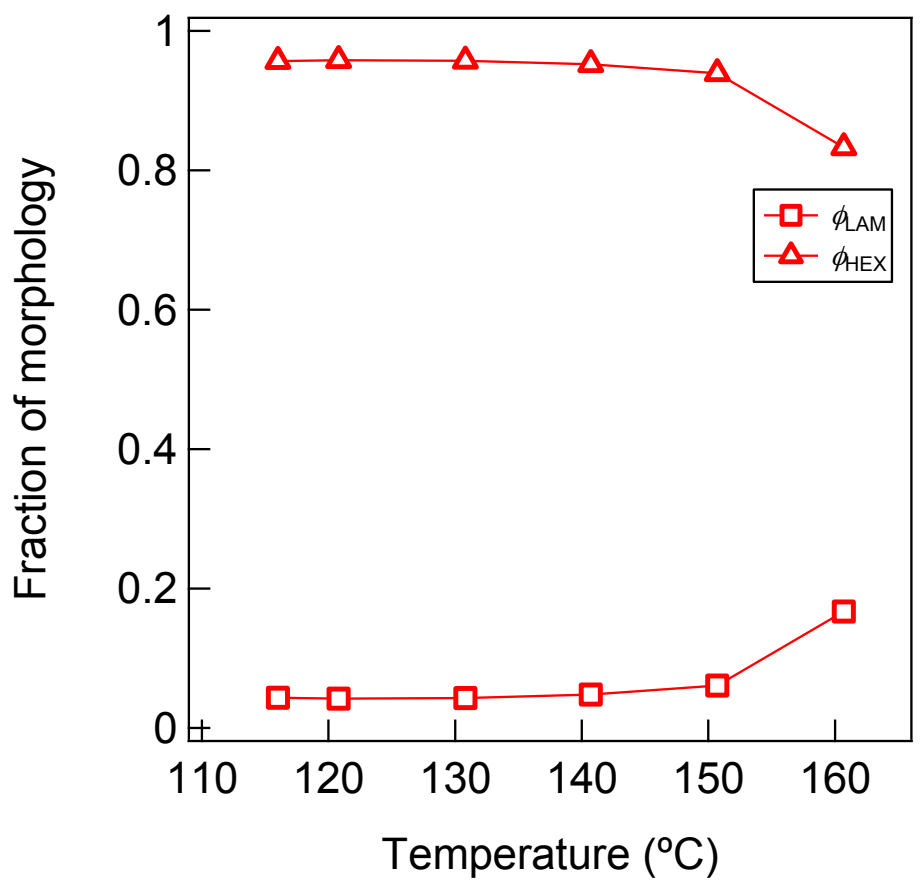

Figure S7. Analysis of ordered-state SAXS data for a blend containing $\phi_{\mathrm{H}}=0.7$ doped with LiTFSI at a molar ratio $r=[\mathrm{Li}] /[\mathrm{EO}]=0.06$. The relative fraction of each phase was determined by comparing the relative intensity of the primary scattering peak of each phase. Lines have been drawn to guide the eye. 


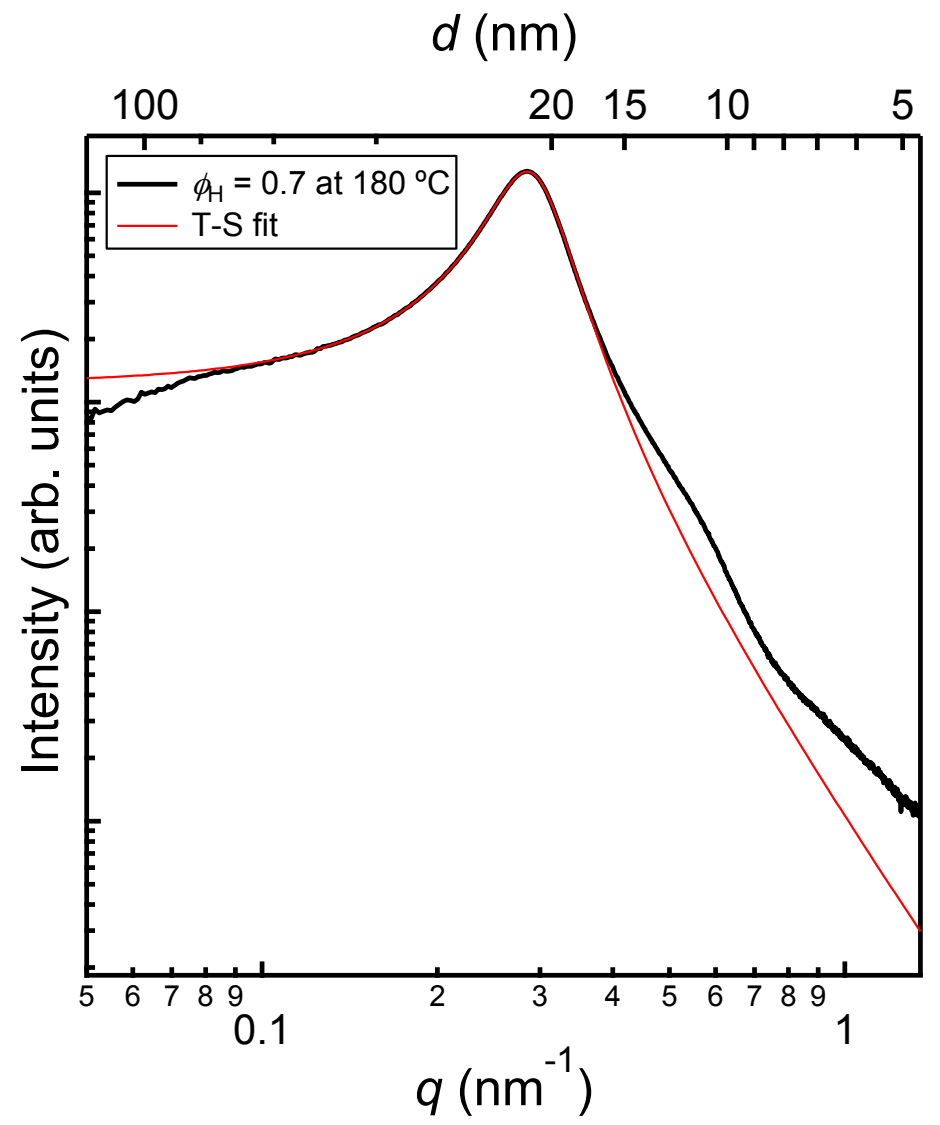

Figure S8. Example of a fit of the Teubner-Strey model to background-subtracted SAXS data for blends containing $\phi_{\mathrm{H}}=0.7$ at $180^{\circ} \mathrm{C}$. 


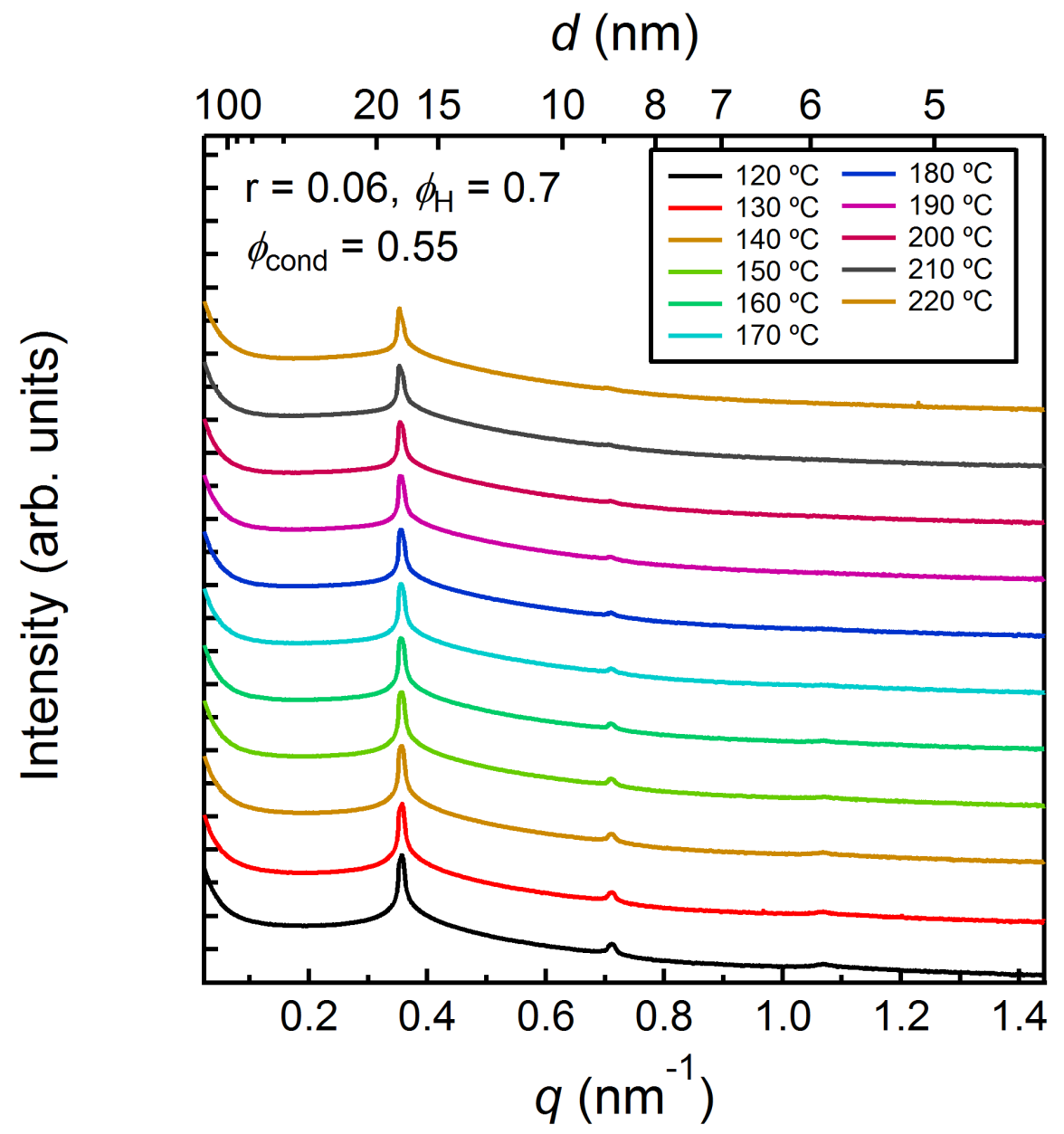

Figure S9. Small angle X-ray scattering traces for a blend containing $\phi_{\mathrm{H}}=0.7$ and $\phi_{\text {cond }}=0.55$ doped with LiTFSI at a molar ratio $r=[\mathrm{Li}] /[\mathrm{EO}]=0.06$. 
(a)

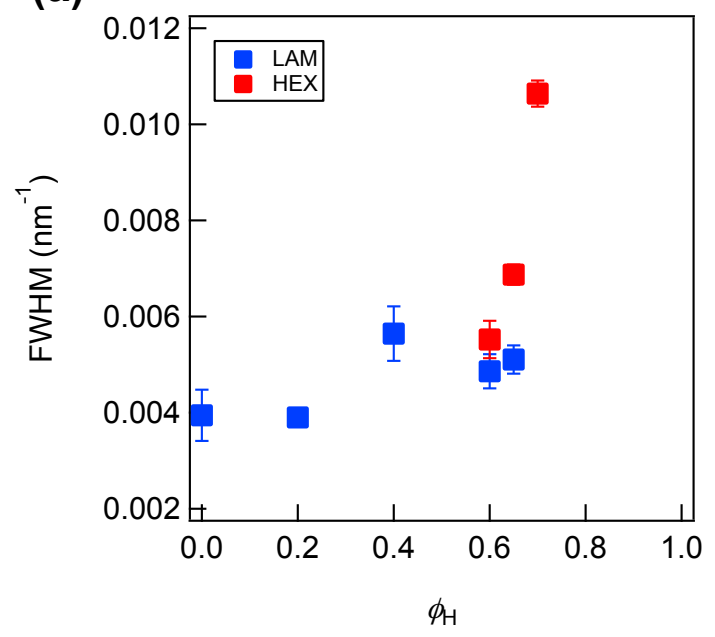

(b)

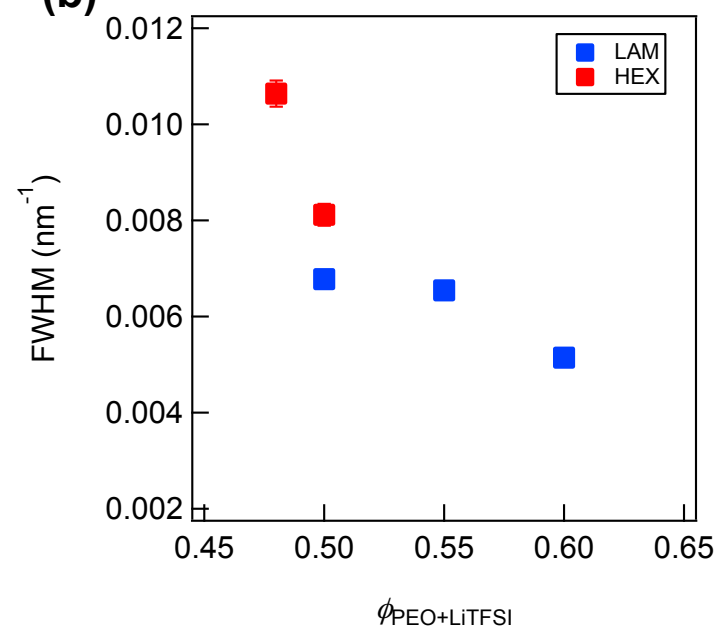

Figure S10. Full-width at half-maximum (FWHM) analysis of primary scattering peaks for (a) samples at $120{ }^{\circ} \mathrm{C}$ as a function of homopolymer loading (data in Figure 3a) and (b) samples at $120{ }^{\circ} \mathrm{C}$ with $\phi_{\mathrm{H}}=0.7$ with different fraction of $\phi_{\mathrm{PEO}+\mathrm{LiTFS}}$ relative to $\phi_{\mathrm{PS}}$ (data in Figure 8). FWHM was determined by applying Lorentzian fits to the peaks. An example of the fitting is shown in Figure S10. Error bars represent one standard deviation. Note that FWHM analysis of the LAM peak for $\phi_{\mathrm{H}}=0.7$ could not be accurately conducted due to the low scattered intensity. 

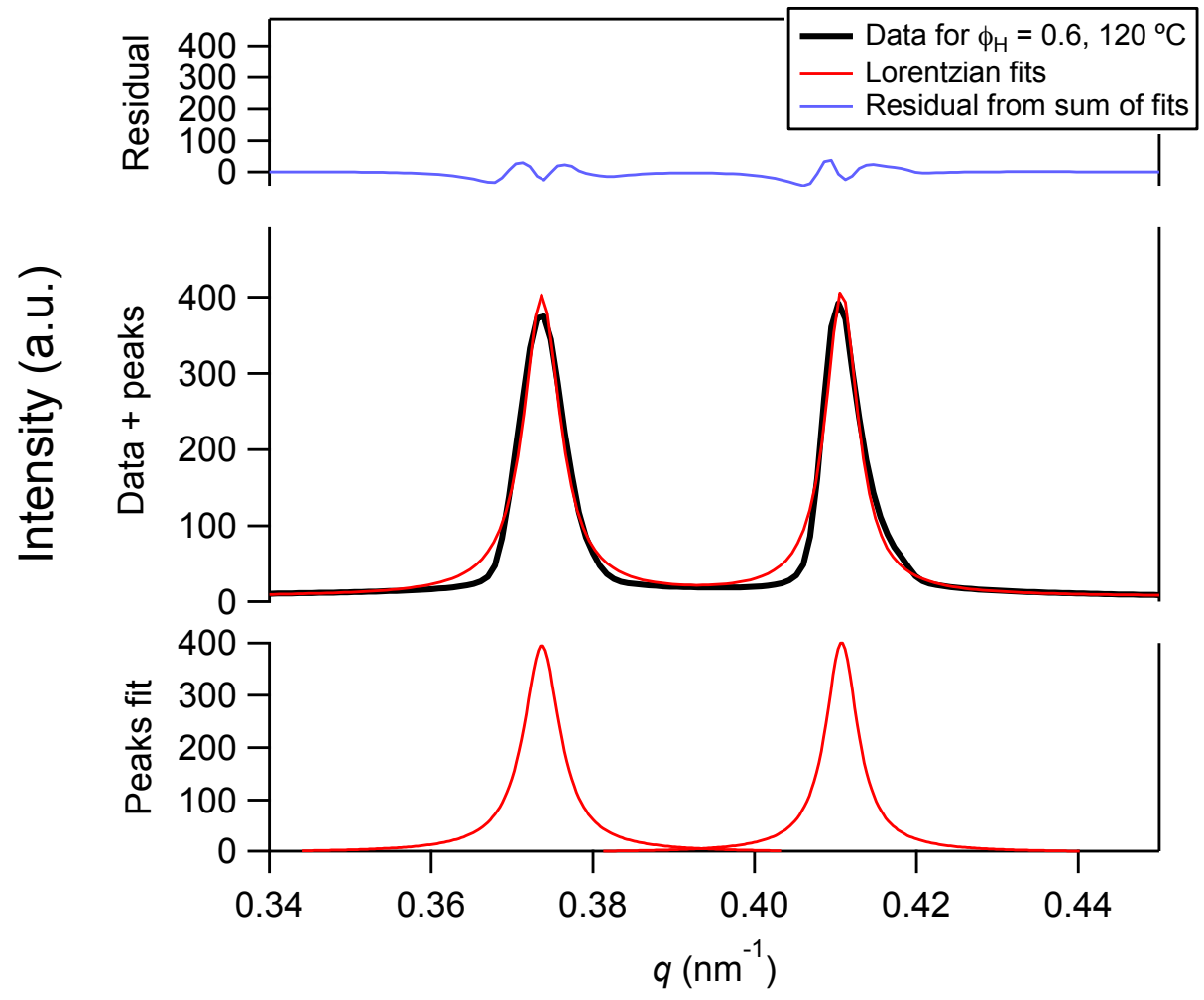

Figure S11. Example of Lorentzian fits used to determine the FWHM shown in Figure S10. The $q^{*}$ peak for each morphology was fit with a single Lorentzian function, and the final fit was a linear combination of the two fitting functions. The residual (top) indicates minimal error with this fitting procedure. 